\title{
Treg/Th17 imbalance is associated with cardiovascular complications in uremic patients undergoing maintenance hemodialysis
}

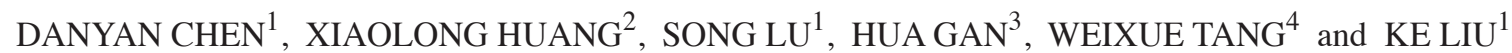 \\ ${ }^{1}$ Department of Endocrinology and Nephrology, The Chongqing Zhongshan Hospital, Chongqing 400013; \\ ${ }^{2}$ Department of Neurosurgery, No. 324 Hospital of PLA, Chongqing 400028; ${ }^{3}$ Department of Nephrology, \\ The First Affiliated Hospital of Chongqing Medical University; ${ }^{4}$ Department of Pathophysiology, \\ Basic Medical College, Chongqing Medical University, Chongqing 400016, P.R. China
}

Received November 28, 2012; Accepted January 14, 2013

DOI: $10.3892 /$ br.2013.63

\begin{abstract}
Investigations of Treg/Th17 imbalance associated with cardiovascular complications in hemodialysis are limited. The aim of this study was to examine the association between Treg/Th17 balance and cardiovascular comorbidity in maintenance hemodialysis (MHD). Uremic patients included in the present study were divided into three groups: the WHD group comprising 30 patients with no cardiovascular complications or maintenance hemodialysis (MHD), the MHD1 group comprising 36 patients presenting with cardiovascular complications during MHD, and the MHD2 group comprising 30 patients with a lack of cardiovascular complications during MHD. The control group comprised 20 healthy volunteers. Th17 and Treg cells were measured by fluorescence-activated cell scanning (FACS). IL-6 and IL-10 levels were determined by enzyme-linked immunosorbent assay (ELISA). Monocyte surface expression of the costimulatory molecules CD80 and CD86 was assessed by FACS after the monocytes were cocultured with Th17 or Treg cells in the presence or absence of IL-17. Results revealed that the percentage of Th17 of total CD4(+) cells was significantly higher in the MHD1 (36.27 $\pm 9.62 \%$ in) and WHD (35.98 $\pm 8.85 \%)$ groups compared with the MHD2 $(19.64 \pm 5.97 \%)$ and healthy $(1.12 \pm 1.52 \%)$ groups. Elevated IL-6 levels were obtained in Th17 cells for the MHD1 and WHD groups, whereas a marked decrease was evident when IL-17 was blocked. However, no significant differences or cardiovascular complications were detected in the expression of CD80 and CD86 in the MHD group, whereas the expression of the uremic subgroups was statistically higher compared with the
\end{abstract}

Correspondence to: Dr Danyan Chen, Department of Endocrinology and Nephrology, The Chongqing Zhongshan Hospital, No. 312 Zhongshan 1st Road, Yu Zhong District, Chongqing 400013, P.R. China

E-mail: clioyoyo@163.com

Key words: cardiovascular, Th17 cell, regulatory $\mathrm{T}$ cell, inflammatory cytokine, costimulatory molecule healthy controls. To the best of our knowledge, this is the first study to demonstrate that the Treg/Th17 imbalance may be associated with the pathogenesis of cardiovascular complications in uremic patients undergoing hemodialysis through the B7-independent upregulation of IL-6 induced by IL-17.

\section{Introduction}

Cardiovascular complications are likely to occur at an early stage in uremic patients undergoing hemodialysis $(1,2)$. Common risk factors, such as hypertension, rarely account for this phenomenon $(3,4)$.

Activation of inflammatory response centered on the excretion of cytokines is closely associated with cardiovascular complications in uremic cases $(5,6)$, particularly by $\mathrm{T}$ cells (7). IL-10-producing $\mathrm{CD} 4{ }^{+} \mathrm{CD} 25^{+} \mathrm{Foxp}^{+}$regulatory $\mathrm{T}$ cells (Tregs) contribute to anti-inflammatory response (8), while the IL-17-induced T-helper cells (Th17s) exhibit a pro-inflammatory role (9). IL-10 and IL-6 play a pivotal role in altering the Treg/Th17 balance. The aim of this study was to examine the association between Treg/Th17 balance and cardiovascular comorbidity in maintenance hemodialysis (MHD).

\section{Subjects and methods}

Patients. This study conformed to the approved institutional guidelines of the Ethics Committee of our Hospital. Informed consent in accordance with the Declaration of Helsinki protocol was obtained from each participant. Of the 96 patients diagnosed with uremia from July, 2010 to January, 2011, 30 cases (18 men and 12 women, mean age $50.33 \pm 16.12$ years) presented with no history of cardiovascular complications (WHD) nor underwent MHD, while the remaining 66 cases (40 men and 26 women, mean age 53.02 \pm 12.73 years) had undergone MHD for at least 3 months, with a mean duration of dialysis of $30.28 \pm 21.24$ months.

The exclusion criteria for this study were: i) the presence of preceding infection, connective tissue diseases, malignant tumors, progressive hepatopathy, hepatitis B, hepatitis C, AIDS, valvular heart disease, as well as bleeding and 
coagulation dysfunction; ii) installation of heart pacemakers; iii) the administration of steroids or non-steroidal antiinflammatory drugs and; iv) the serum level of parathyroid hormone $(\mathrm{PTH}) \geq 400 \mathrm{pg} / \mathrm{ml}$. The underlying diseases of the WHD group included chronic glomerulonephritis $(n=12)$, hypertensive nephrosclerosis $(n=7)$, diabetic nephropathy $(n=4)$, polycystic kidney $(n=2)$, chronic interstitial nephritis $(n=3)$ and 2 cases with unknown origin, while those of the MHD group included 24 cases with chronic glomerulonephritis, 16 with hypertensive nephrosclerosis, 10 with diabetic nephropathy, 6 with chronic interstitial nephritis, 4 with polycystic kidney, 4 with cerebrovascular disease and 2 with unknown origin. A total of 66 cases of MHD were further divided depending on presence of cardiovascular diseases. Consequently, 36 cases (MHD1) were identified with unstable angina $(n=6)$, left ventricular hypertrophy $(n=10)$, severe arrhythmia $(n=14)$, congestive heart failure $(n=6)$, while no cardiovascular diseases were identified in the remaining 30 cases (MHD2). Moreover, 20 healthy individuals (10 men and 10 women, mean age $43.89 \pm 8.72$ years) comprised the control group $(\mathrm{CON})$. Patient data were collected for statistical analysis including age, gender, duration of dialysis, smoking, history of diabetes mellitus, hyperlipidaemia, blood pressure, body mass index (BMI), laboratory and echocardiographical parameters, as well as medications. BMI was calculated as height/weight ${ }^{2}$.

Hemodialysis strategy. Hemodialysis patients in the MHD group were treated using polysulfone membranes, with heparin serving as an anticoagulant. The hemodialysis blood flow rate was set at $200-250 \mathrm{ml} / \mathrm{min}$, while the dialysate flow rate was $500 \mathrm{ml} / \mathrm{min}$. Hemodialysis was conducted at a regular frequency of 2-3 times per week, lasting 4-5 h each time.

Peripheral mononuclear cell (PMNC) preparation. Fresh serum samples were obtained from the ulnar vein on an empty stomach from all the patients and healthy volunteers. Samples were collected in tubes containing heparin. The levels of haemoglobin, albumin, serum creatinine, blood urea nitrogen (BUN), lipid, glucose, PTH and CK-MB in the blood were measured. PMNCs were purified by Ficoll-Hypaque (Sigma Chemical, St. Louis, MO, USA). Cells were separated using density-gradient centrifugation and cultured in RPMI-1640 medium (Gibco-BRL Life Technologies, Merelbeke, Belgium) supplemented with 10\% FCS (Hyclone, Logan, UT, USA), $2 \mathrm{mM}$ L-glutamine, $100 \mathrm{U} / \mathrm{ml}$ penicillin, and $100 \mu \mathrm{g} / \mathrm{ml}$ streptomycin, in a humidified incubator with $5 \% \mathrm{CO}_{2}$ at $37^{\circ} \mathrm{C}$ (Shellab 2306, USA).

Flow cytometry. Flow cytometry and cell sorting were performed on the BD FACSAria Cell-Sorting System (BD Biosciences, San Jose, CA, USA). Separated cells were washed with FACS buffer (phosphate-buffered saline supplemented with $0.1 \%$ sodium azide and $2 \%$ fetal bovine serum), fixed with $4 \%$ paraformaldehyde for $10 \mathrm{~min}$, and permeabilized with $0.1 \%$ Triton X-100. The cells were incubated with anti-CD4, anti-CD80 or anti-CD86 conjugated with PE, as well as anti-CD25 and Anti-Foxp3 labeled with FITC (Santa Cruz Biotechnology, Inc., Santa Cruz, CA, USA) according to the manufacturer's instructions. The cells were then washed with the FACS buffer and underwent flow cytometric analysis with CellQuest Pro (BD Biosciences). The results were presented as the geometric mean fluorescence intensity.

Magnetic bead cell sorting. Using CD4 and CD25 microbead selection (Miltenyi Biotec, Auburn, CA, USA), Treg cells were obtained as $\mathrm{CD} 4^{+} \mathrm{CD} 25^{+}$and Th17 cells as $\mathrm{CD} 4^{+} \mathrm{CD} 25^{-}$, as previously described $(10,11)$. Similarly, peripheral monocytes, isolated from PMNC using $\mathrm{CD} 14^{+}$microbead selection (Miltenyi Biotec), were respectively seeded into an anti-CD3-coated round-bottomed 96-well plate at $1 \times 10^{5} /$ well in $200 \mu \mathrm{l}$ and subdivided into four subgroups: i) exclusive culture of $\mathrm{CD} 14^{+}$cells; ii) coculture with $\mathrm{CD} 4^{+} \mathrm{CD} 25^{+}$ $\mathrm{T}$ cells at a ratio of $1: 1$; iii) coculture with $\mathrm{CD} 4^{+} \mathrm{CD} 25^{-} \mathrm{T}$ cells at a ratio of $1: 1$ in the absence of anti-IL-17Ab and; iv) coculture with $\mathrm{CD} 4^{+} \mathrm{CD} 25^{-} \mathrm{T}$ cells at a ratio of $1: 1 \mathrm{in}$ the presence of anti-IL-17Ab. Cells were cultured for 3 days at $37^{\circ} \mathrm{C}$ in RPMI-1640 medium. Experiments were repeated three times.

Cytokine secretion. The supernatants of cultured systems were collected and stored at $-80^{\circ} \mathrm{C}$ for subsequent experiments. Prior to use, samples were treated for $30 \mathrm{~min}$ at $37^{\circ} \mathrm{C}$ with $25 \mathrm{U} / \mathrm{ml}$ of hyaluronidase (Sigma-Aldrich, Zwijndrecht, The Netherlands). Soluble IL-6 and IL-10 levels were measured by enzyme-linked immunosorbent assay (ELISA) using the commercially available kits (R\&D Systems, Minneapolis, MN, USA). Optical densities were measured at $450 \mathrm{~nm}$ (Spectra II; SLT, Vienna, Austria) and cytokine concentrations were determined from the optical density value according to standard curves.

Statistical analysis. Data were assessed using the SPSS software 16.0 (Statistical Package for the Social Sciences, version 13.0; SPSS Inc., Chicago, IL, USA). The two-tailed unpaired Student's t-test was used for comparison between groups and subgroups. $\mathrm{P}<0.05$ was considered to indicate a statistically significant difference.

\section{Results}

Subjects. No significant differences were found among the MHD1, MHD2 and WHD groups with regards to characteristics such as age, gender, risk factors, therapeutic regimens, ejection factor, left ventricular end-diastolic diameter and left ventricular end-systolic diameter (LVEDD and LVESD) ( $>0.05)$. The levels of BUN and serum creatinine were significantly higher in the WHD group when compared with the MHD1 and MHD2 groups undergoing hemodialysis $(\mathrm{P}<0.05)$. In the MHD1 group, the left ventricular mass index of $189.00 \pm 35.38$ was the highest, followed by $120.05 \pm 2.59$ in the WHD and $102.34 \pm 11.30$ in the MHD2 groups $(\mathrm{P}<0.05)$. In the MHD2 group, the fractional shortening ratio of $33.79 \pm 2.08$ was the highest, compared to $31.72 \pm 3.39$ in the WHD and $26.86 \pm 1.82$ in the MHD1 groups $(\mathrm{P}<0.05)$. No significant differences were found in the traditional risk factors of atherosclerotic cardiovascular disease, such as smoking, hypertension, diabetes mellitus, hyperlipidaemia, anemia, left ventricular hypertrophy, and increased age, as well as other baseline characteristics among in any of the uremic subjects (Table I). 
Table I. Baseline characteristics of the study population.

\begin{tabular}{|c|c|c|c|c|}
\hline Characteristics & $\mathrm{CON}(\mathrm{n}=20)$ & WHD (n=30) & MHD1 (n=36) & MHD2 (n=30) \\
\hline Age (years) & $43.89 \pm 8.72$ & $50.33 \pm 16.12$ & $55.70 \pm 12.64$ & $46.85 \pm 11.02$ \\
\hline Gender (male:female) & $10: 10$ & $18: 12$ & $24: 12$ & $16: 14$ \\
\hline Months since initiating dialysis & - & - & $30.28 \pm 23.04$ & $30.30 \pm 17.20$ \\
\hline \multicolumn{5}{|l|}{ Risk factors } \\
\hline Smoking, n (\%) & $4(20)$ & $10(33)$ & $11(31)$ & $8(27)$ \\
\hline Diabetes mellitus, n (\%) & $0(0)$ & $4(13)$ & $6(17)$ & $4(13)$ \\
\hline Hyperlipidaemia, n (\%) & $2(10)$ & $11(37)$ & $12(33)$ & $8(27)$ \\
\hline Systolic blood pressure (mmHg) & $120.44 \pm 8.88$ & $147.14 \pm 16.15$ & $149.10 \pm 15.60$ & $141.15 \pm 16.88$ \\
\hline Diastolic blood pressure (mmHg) & $72.94 \pm 6.48$ & $88.52 \pm 15.55$ & $83.70 \pm 9.83$ & $86.31 \pm 9.22$ \\
\hline Body mass index & $20.99 \pm 1.81$ & $22.54 \pm 2.43$ & $21.75 \pm 3.39$ & $22.09 \pm 1.71$ \\
\hline Haemoglobin $(\mathrm{g} / \mathrm{l})$ & $130.56 \pm 11.08$ & $93.40 \pm 21.90$ & $93.63 \pm 22.81$ & $105.62 \pm 14.14$ \\
\hline Albumin $(g / l)$ & $37.47 \pm 5.56$ & $32.57 \pm 5.06$ & $35.47 \pm 4.61$ & $37.69 \pm 6.64$ \\
\hline \multicolumn{5}{|l|}{ Other laboratory parameters } \\
\hline Blood urea nitrogen $(\mathrm{mmol} / \mathrm{l})$ & $5.86 \pm 0.90$ & $31.16 \pm 10.59$ & $22.68 \pm 8.94^{\mathrm{a}}$ & $20.26 \pm 8.91^{\mathrm{a}}$ \\
\hline Serum creatinine $(\mu \mathrm{mol} / 1)$ & $89.5 \pm 15.21$ & $997.10 \pm 325.25$ & $728.54 \pm 288.06^{\mathrm{a}}$ & $710.88 \pm 249.38^{\mathrm{a}}$ \\
\hline \multicolumn{5}{|l|}{ Echocardiography } \\
\hline Left ventricular systolic lumen (mm) & $31.33 \pm 2.99$ & $34.00 \pm 4.68$ & $34.97 \pm 2.58$ & $31.77 \pm 2.28$ \\
\hline Left ventricular diastolic lumen (mm) & $43.39 \pm 3.70$ & $43.64 \pm 5.06$ & $49.70 \pm 3.39$ & $42.91 \pm 4.56$ \\
\hline Ejection fraction $(\%)$ & $58.82 \pm 2.72$ & $57.73 \pm 4.69$ & $57.77 \pm 7.68$ & $58.95 \pm 3.21$ \\
\hline Fractional shortening $(\%)$ & $37.27 \pm 4.73$ & $31.72 \pm 3.39$ & $26.86 \pm 1.82^{\mathrm{a}}$ & $33.79 \pm 2.08^{\mathrm{b}}$ \\
\hline Left ventricular mass index $\left(\mathrm{g} / \mathrm{m}^{2}\right)$ & $84.71 \pm 6.94$ & $120.05 \pm 2.59^{b}$ & $189.00 \pm 35.38^{\mathrm{a}}$ & $102.34 \pm 11.30^{\mathrm{a}, \mathrm{b}}$ \\
\hline \multicolumn{5}{|l|}{ Medications } \\
\hline rHu-EPO, n (\%) & $0(0)$ & $18(60)$ & $22(61)$ & $20(67)$ \\
\hline$\beta$-blockers, n (\%) & $0(0)$ & $9(30)$ & $10(28)$ & $10(33)$ \\
\hline Statins, n $(\%)$ & $2(10)$ & $9(30)$ & $12(33)$ & $8(27)$ \\
\hline
\end{tabular}

CON, the healthy control group; WHD, the group with neither cardiovascular complications nor maintenance hemodialysis; MHD, maintenance hemodialysis; MHD1, the group presenting with cardiovascular complications during MHD; MHD2, the group lack of cardiovascular complications during MHD; rHu-EPO, recombinant human erythropoietin. Values are presented as teh mean \pm standard deviation or number. ${ }^{\mathrm{a}} \mathrm{P}<0.05 \mathrm{WHD}$ vs. MHD, ${ }^{\mathrm{b}} \mathrm{P}<0.05 \mathrm{MHD} 1$ vs. MHD2.

Table II. Comparison of Treg, Th17 frequencies and Treg/Th17 ratios among groups (mean \pm standard deviation).

\begin{tabular}{lrrrr}
\hline & \multicolumn{1}{c}{ MHD1 } & MHD2 & WHD & CON \\
\hline Treg $(\%)$ & $5.05 \pm 2.38^{\mathrm{a}}$ & $6.06 \pm 1.79^{\mathrm{a}}$ & $5.92 \pm 3.29^{\mathrm{a}}$ & $0.32 \pm 0.44$ \\
Th17 (\%) & $36.27 \pm 9.62^{\mathrm{a}}$ & $19.64 \pm 5.97^{\mathrm{a}, \mathrm{b}, \mathrm{c}}$ & $35.98 \pm 8.85^{\mathrm{a}}$ & $1.12 \pm 1.52$ \\
Treg/Th17 & $14.43 \pm 8.28^{\mathrm{a}}$ & $21.21 \pm 10.28^{\mathrm{a}, \mathrm{b}, \mathrm{c}}$ & $15.35 \pm 4.86^{\mathrm{a}}$ & $26.31 \pm 2.26$ \\
\hline
\end{tabular}

MHD, maintenance hemodialysis; MHD1, the group presenting with cardiovascular complications during MHD; MHD2, the group lacking cardiovascular complications during MHD; WHD, the group with neither cardiovascular complications nor maintenance hemodialysis; CON, the healthy control group; Treg, regulatory T cell; Th17, IL-17-induced T-helper cell. ${ }^{a} \mathrm{P}<0.05$ vs. CON, ${ }^{b} \mathrm{P}<0.05 \mathrm{WHD}$ vs. MHD, ${ }^{\mathrm{c}} \mathrm{P}<0.05$ MHD1 vs. MHD2.

Enhanced Th17 proliferation and reversal of the Treg/Th17 ratio in uremic patients, particularly for the WHD and MHDI groups. As shown in Table II and Fig. 1, the frequencies of Treg and Th17 cells were significantly higher in the uremic groups when compared with the CON. By contrast, the ratio of
Treg/Th17 was lower in the uremic groups compared with the $\mathrm{CON}(\mathrm{P}<0.05)$. No significant differences of the Treg frequencies were identified among the WHD, MHD1 and MHD2 subgroups $(\mathrm{P}>0.05)$. The WHD subgroup had a markedly higher frequency of Th17 cells and a notably lower Treg/Th17 
A

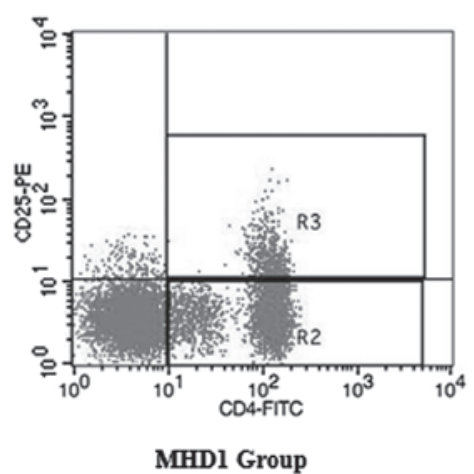

C

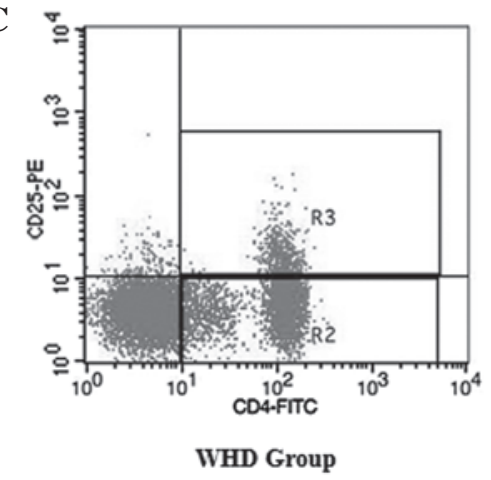

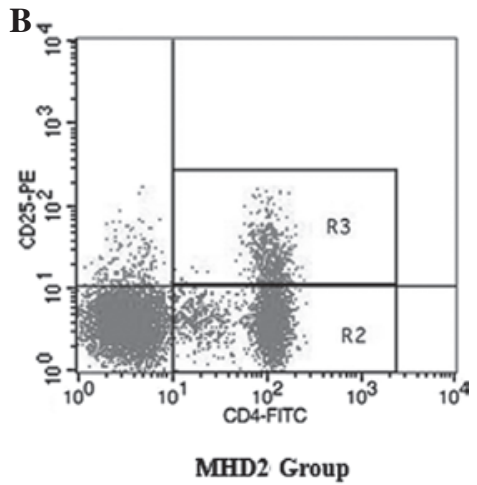

D

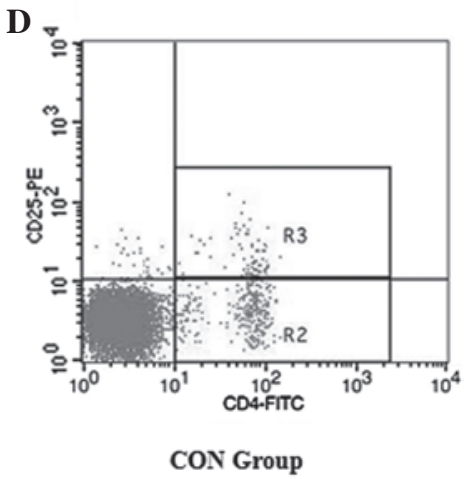

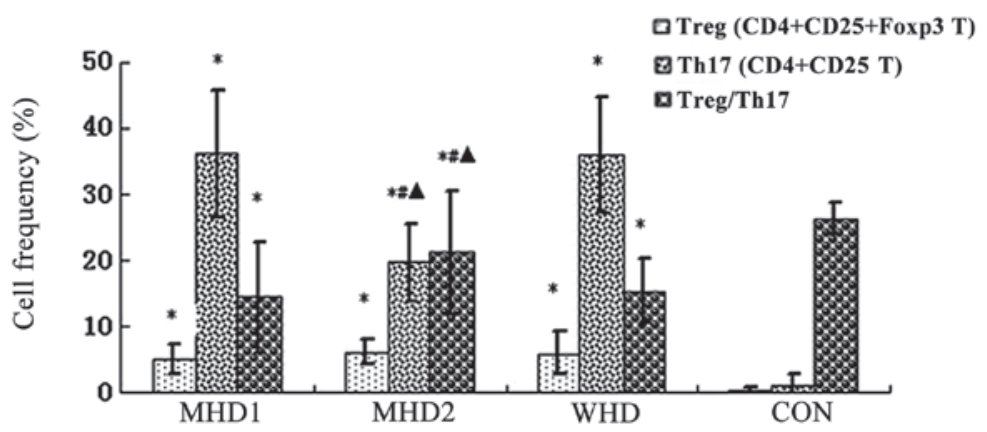

Figure 1. As detected by flow cytometry, $\mathrm{CD} 4{ }^{+} \mathrm{CD} 25^{+}$populations were counted as Treg cells, while $\mathrm{CD} 4^{+} \mathrm{CD} 25^{-}$populations were counted as Th17 cells (A) Frequencies of Treg and Th17 cells in the MHD1, (B) MHD2, (C) WHD and CON groups. As shown in (E), compared to the control group, the frequencies of Treg and Th17 cells were significantly higher and the ratio of Treg/Th17 was lower in the uremic groups $(\mathrm{P}<0.05)$, compared to the MHD2 group. The WHD and MHD1 subgroups had markedly higher frequencies of Th17 cells and a notably lower Treg/Th17 ratios $(\mathrm{P}<0.05) .{ }^{*} \mathrm{P}<0.05$, compared with the CON group; ${ }^{*} \mathrm{P}<0.05$,

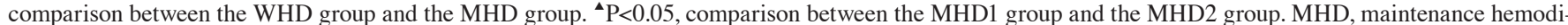
alysis; MHD1, group presenting with cardiovascular complications during MHD; MHD2, group lacking cardiovascular complications during MHD; WHD, group with neither cardiovascular complications nor maintenance hemodialysis, CON, healthy control group; Treg, regulatory T cell; Th17; IL-17-induced T-helper cell

Table III. IL-6 concentration in cocultured supernatants by ELISA.

\begin{tabular}{lcccc}
\hline IL-6 (pg/ml) & CD14 monocytes & $\begin{array}{c}\text { Monocyte-Treg } \\
\text { cocultures }\end{array}$ & $\begin{array}{c}\text { Monocyte-Th17 } \\
\text { cocultures }\end{array}$ & $\begin{array}{c}\text { Monocyte-Th17 cocultures } \\
\text { in the presence of anti-IL-17 }\end{array}$ \\
\hline MHD1 (n=36) & $75.04 \pm 3.25^{\mathrm{a}}$ & $56.12 \pm 1.49^{\mathrm{a}}$ & $82.74 \pm 3.28^{\mathrm{a}, \mathrm{d}}$ & $57.71 \pm 2.87^{\mathrm{a}, \mathrm{b}}$ \\
MHD2 (n=30) & $44.17 \pm 4.75^{\mathrm{a}, \mathrm{c}}$ & $35.15 \pm 0.72^{\mathrm{a}, \mathrm{c}}$ & $49.58 \pm 4.26^{\mathrm{a}, \mathrm{c}}$ & $37.02 \pm 1.22^{\mathrm{a}, \mathrm{c}}$ \\
WHD (n=30) & $78.94 \pm 5.44^{\mathrm{a}}$ & $61.18 \pm 1.36^{\mathrm{a}}$ & $88.94 \pm 1.83^{\mathrm{a}, \mathrm{d}}$ & $64.47 \pm 1.98^{\mathrm{a}, \mathrm{e}}$ \\
CON (n=20) & $15.56 \pm 1.58$ & $14.68 \pm 1.66$ & $16.98 \pm 1.25$ & $16.04 \pm 0.83$ \\
\hline
\end{tabular}

MHD, maintenance hemodialysis; MHD1, the group presenting with cardiovascular complications during MHD; MHD2, the group lack of cardiovascular complications during MHD; WHD, the group with neither cardiovascular complications nor maintenance hemodialysis; CON, the healthy control group; Treg, regulatory T cell; Th17, IL-17-induced T-helper cell; IL, interleukin; CD, cluster of differentiation. ${ }^{a} \mathrm{P}<0.05$, compared with the CON group, ${ }^{b} \mathrm{P}<0.05$, comparison between the WHD group and the MHD group, ${ }^{\mathrm{C}} \mathrm{P}<0.05$, comparison between the MHD1 group and the MHD2 group, ${ }^{\mathrm{d}} \mathrm{P}<0.05$, comparison between the moncyte-Treg and monocyte-Th 17 cocultures, ${ }^{\mathrm{e}} \mathrm{P}<0.05$, comparison between the the moncyte-Th17 cocultures in the absence or presence of anti-IL-17. 
Table IV. IL-10 concentration in cocultured supernatants by ELISA.

\begin{tabular}{lcccc}
\hline IL-10 $(\mathrm{pg} / \mathrm{ml})$ & CD14 ${ }^{+}$monocytes & $\begin{array}{c}\text { Monocyte-Treg } \\
\text { cocultures }\end{array}$ & $\begin{array}{c}\text { Monocyte-Th17 } \\
\text { cocultures }\end{array}$ & $\begin{array}{c}\text { Monocyte-Th17 cocultures } \\
\text { in the presence of anti-IL-17 }\end{array}$ \\
MHD1 (n=36) & $12.62 \pm 1.90^{\mathrm{a}, \mathrm{b}}$ & $17.47 \pm 1.62^{\mathrm{a}, \mathrm{b}}$ & $19.92 \pm 2.53^{\mathrm{a}, \mathrm{b}}$ & $17.52 \pm 2.71^{\mathrm{a}, \mathrm{b}}$ \\
MHD2 (n=30) & $21.75 \pm 1.17$ & $23.29 \pm 2.36$ & $23.48 \pm 1.35$ & $23.22 \pm 2.11$ \\
WHD (n=30) & $11.27 \pm 2.22^{\mathrm{a}, \mathrm{b}}$ & $13.63 \pm 2.45^{\mathrm{a}, \mathrm{b}}$ & $18.58 \pm 1.91^{\mathrm{a}, \mathrm{b}}$ & $15.36 \pm 1.18^{\mathrm{a}, \mathrm{b}}$ \\
CON (n=20) & $26.94 \pm 0.54$ & $24.15 \pm 1.50$ & $27.62 \pm 0.68$ & $23.77 \pm 2.31$ \\
\hline
\end{tabular}

MHD, maintenance hemodialysis; MHD1, the group presenting with cardiovascular complications during MHD; MHD2, the group lacking cardiovascular complications during MHD; WHD, the group with neither cardiovascular complications nor maintenance hemodialysis; CON, the healthy control group; Treg, regulatory T cell; Th17, IL-17-induced T-helper cell; IL, interleukin; CD, cluster of differentiation; ELISA, enzyme-linked immunosorbent assay. ${ }^{a} \mathrm{P}<0.05$, compared with the $\mathrm{CON}$ group; ${ }^{\mathrm{b}} \mathrm{P}<0.05$, compared with the MHD2 group.

Table V. Expression of costimulatory molecules CD80 and CD86 on monocytes when cocultured with Treg or Th17 cells in the absense or presense of anti-IL-17.

\begin{tabular}{|c|c|c|c|c|}
\hline & $\mathrm{CD}_{14}{ }^{+}$monocytes & $\begin{array}{l}\text { Monocyte-Treg } \\
\text { cocultures }\end{array}$ & $\begin{array}{l}\text { Monocyte-Th17 } \\
\text { cocultures }\end{array}$ & $\begin{array}{l}\text { Monocyte-Th17 cocultures } \\
\text { in the presence of anti-IL-17 }\end{array}$ \\
\hline \multicolumn{5}{|l|}{ CD80 } \\
\hline MHD1 & $53.32 \pm 0.75^{\mathrm{a}}$ & $51.29 \pm 1.28^{\mathrm{a}}$ & $57.14 \pm 1.07^{\mathrm{a}}$ & $54.00 \pm 0.79^{\mathrm{a}}$ \\
\hline MHD2 & $49.55 . \pm 0.72^{\mathrm{a}}$ & $47.85 \pm 0.92^{\mathrm{a}}$ & $52.86 . \pm 0.18^{a}$ & $50.22 \pm 1.10^{\mathrm{a}}$ \\
\hline WHD & $59.50 \pm 0.62^{\mathrm{a}}$ & $56.76 \pm 1.65^{\mathrm{a}}$ & $66.70 \pm 1.54^{\mathrm{a}}$ & $61.29 \pm 1.59^{\mathrm{a}}$ \\
\hline $\mathrm{CON}$ & $23.53 \pm 2.52$ & $23.50 \pm 1.45$ & $27.97 \pm 0.27$ & $25.58 \pm 0.45$ \\
\hline \multicolumn{5}{|l|}{ CD86 } \\
\hline MHD1 & $42.98 \pm 0.31^{\mathrm{a}}$ & $40.82 \pm 1.51^{\text {as }}$ & $47.21 \pm 0.56^{\mathrm{a}}$ & $43.73 \pm 0.37^{\mathrm{a}}$ \\
\hline MHD2 & $40.58 \pm 0.54^{\mathrm{a}}$ & $39.42 \pm 0.36^{\mathrm{a}}$ & $43.56 . \pm 0.97^{\mathrm{a}}$ & $42.11 \pm 0.04^{\mathrm{a}}$ \\
\hline WHD & $47.93 \pm 0.33^{\mathrm{a}}$ & $44.88 \pm 1.18^{\mathrm{a}}$ & $50.44 \pm 1.63^{\mathrm{a}}$ & $46.83 \pm 1.02^{\mathrm{a}}$ \\
\hline $\mathrm{CON}$ & $23.06 \pm 1.40$ & $23.02 \pm 1.48$ & $28.06 \pm 0.50$ & $25.56 \pm 1.34$ \\
\hline
\end{tabular}

MHD, maintenance hemodialysis; MHD1, the group presenting with cardiovascular complications during MHD; MHD2; the group lacking cardiovascular complications during MHD; WHD, the group with neither cardiovascular complications nor maintenance hemodialysis; CON, the healthy control group; Treg, regulatory T cell; Th17, IL-17-induced T-helper cell; IL, interleukin; CD, cluster of differentiation. ${ }^{a} \mathrm{P}<0.05$, compared with the $\mathrm{CON}$ group.

ratio compared with the MHD2 subgroup $(\mathrm{P}<0.05)$. Between the MHD subgroups, the Th17 frequencies of the MHD1 subgroup were notably higher, while the Treg/Th17 ratio was notably lower compared with the MHD2 group $(\mathrm{P}<0.05)$.

Restoration of Th17-induced IL-6 overproduction by anti-IL-17 in cocultured supernatants of uremic cohort. Expression of IL-6 and IL-10 was detected in the cocultured supernatants. Compared with the healthy controls, IL-6 concentration levels of the subgroups of each uremic group were significantly higher, respectively $(\mathrm{P}<0.05)$ (Table III). By contrast, compared with the MHD2 group, IL-6 concentration levels of the MHD1 and WHD subgroups were significantly higher $(\mathrm{P}<0.05)$. However, no significant differences were observed when comparing the MHD1 group with the WHD group $(\mathrm{P}>0.05)$. Treg cells inhibited, while Th17 cells increased IL- 6 expression in the WHD, MHD1 and
MHD2 subgroups, although no statistically significant differences were detected $(\mathrm{P}>0.05)$. Additionally, in the MHD1 and WHD groups, the addition of anti-IL-17 was found to suppress the upregulation of IL-6 induced by Th17 cells $(\mathrm{P}<0.05)$. By contrast, compared with the healthy or MHD2 groups, the IL-10 concentrations of the cocultured subgroups were significantly decreased in the MHD1 and WHD groups, respectively $(\mathrm{P}<0.05)$ (Table IV).

Mild alteration of costimulatory molecules CD80 and CD86 on monocytes when cocultured with Treg or Th17 cells in the presence or absence of anti-IL-17. To mimic signaling via the B7 family of costimulatory molecules, the monocyte surface expression of the costimulatory molecules CD80 and CD86 was detected by flow cytometry (Table V). When compared with the healthy group, a significantly elevated percentage of monocytes expressing either CD80 or CD86 
was detected in the pathological cohort between the same paired subgroups $(\mathrm{P}<0.05)$. Although the tendency of CD80 and CD86 to induce Treg downregulation and Th17 upregulation was identified, no significant differences were observed $(\mathrm{P}>0.05)$. The addition of anti-IL-17 $\mathrm{mAb}$ to the monocyte-Th17 cocultures partly resulted in the suppression of Th17-induced CD80 and CD86 activation in all the studied subsets, although no statistically significant differences were noted $(\mathrm{P}>0.05)$.

\section{Discussion}

Cardiovascular disease, an inflammatory disorder regulated by $\mathrm{T}$ lymphocytes, is the leading cause of mortality in chronic kidney diseases and the uremic state. One of the risk factors for potential cardiovascular complications in uremic adults is the autoimmune component, which has been characterized by the excretion of inflammatory cytokines (IL-1, IL-6, IL-10 and TNF- $\alpha$ ), particularly by $\mathrm{T}$ cells at an early stage. Homeostasis of uremic patients is disturbed by a complex network of immune T-cell subpopulations. Recently, Treg and Th17 cells, two distinct subsets from Th1 and Th2 cells, have been described to have the opposite effects on autoimmunity (12). Treg/Th17 balance controls inflammation and may therefore be crucial in the pathogenesis of plaque destabilization as well as the onset of acute coronary syndrome, which includes unstable angina and acute myocardial infarction (?). To demonstrate whether Treg/Th17 is associated with cardiovascular disease in uremic patients during MHD, we detected Treg/Th17 functions on different levels including cell frequencies, related cytokine secretion and key costimulatory molecules.

It is noteworthy that the Treg/Th17 cell function disequilibrium might act synergistically with calcification in the high incidence of cardiovascular disease subsequent to hemodialysis, which has been indirectly demonstrated in a previous study with the aid of ex vivo intervention of recombinant human bone morphogenetic protein-2 (13). However, the previous study was completely based on hemodialysis patients, who did not have uremia or were not assessed for baseline cardiovascular disease. Consequently, findings of that study render it difficult to distinguish whether the Treg/Th17 imbalance was mainly affected by uremia-related inflammation, cardiovascular disease-related conditions during hemodialysis or other pathological alterations, such as calcification. Therefore, the present study selected 30 uremic cases with no cardiovascular complications or MHD as the WHD baseline control.

The results of this study indicate that uremic patients, particularly those in the MHD1 and WHD cohort, exhibited significant increases of peripheral Th17 frequencies and Th17-related cytokines (IL-6) levels. Additionally, a mild alteration of Treg frequencies and Treg-related cytokine (IL-10) levels was observed when compared with the healthy cohort. These findings suggest that the disturbed Treg/Th17 numerical and functional balance has a potential role in the uremic cases and in the development of adverse cardiovascular events for this cohort, to some extent, mainly via IL-6-mediated Th17 differentiation rather than Treg. This observation is concordance with those of recent studies which described that the function fo Treg was defective in certain human autoimmune diseases, including multiple sclerosis (14), type 1 diabetes mellitus, and rheumatoid arthritis (15). Consistent with the previous conclusion that Treg cells were able to induce $\mathrm{CD} 4^{+} \mathrm{CD} 25^{-}$naive $\mathrm{T}$ cells or Treg cells themselves to differentiate into Th17 in the presence of IL-6 alone without exogenous TGF- $\beta$ (16), we observed that IL-6 rather than IL-10 might be the key switch immunoregulatory factor contributing to the shift of the Th17/Treg ratio in the uremic cases and in the development of adverse cardiovascular complications for this cohort. Moreover, IL-6 is a pleiotropic cytokine involved in determining the relative balance of inflammation-promoting Th17 vs. anti-inflammatory Treg cells by inducing the development of Th17 cells from naive $\mathrm{T}$ cells. Our results have demonstrated that MHD effectively improved the pathological Treg/Th17 imbalance and IL-6 overproduction for the uremic cohort. These findings suggest that blocking IL-6-induced Th17 differentiation is a promising therapeutic target that is likely to mitigate myocardial inflammation and reduce the risk of adverse cardiovascular events. By contrast, anti-IL-17 significantly restored the Th17-induced IL-6 secretion in uremic cases, particularly for the MHD1 and WHD cohorts, without altering the costimulatory molecules CD80 and CD86 expression on the surface of monocytes. Thus, peripheral blood Th17 likely plays a crucial role in the pathogenesis of cardiovascular complications for uremic patients in a costimulatory molecule independent pattern, although the concrete mechanisms remain unclear. In this study, blocking IL-6 seems to be a more optimal target compared with either CD80 or CD86 in preventing adverse cardiovascular events for uremic patients during MHD.

A larger patient sample and more isolated Treg cells for functional studies should be considered for future studies. Moreover, a workup of Treg/Th17 cytokines, including TGF- $\beta$ and more costimulatory molecules, which were unavailable in our study, may provide a more precise tool for determining the correlation between inflammation status and various cardiovascular complications during hemodialysis.

In summary, our findings provided evidence that Treg/Th17 imbalance plays a potential role in the pathogenesis of cardiovascular complications for uremic patients undergoing hemodialysis. Additionally, blockage of Th17 differentiation activated by IL-17-induced IL-6 overproduction may be an attractive therapeutic goal. However, this remains to be proven in a larger scale patient cohort. The precise mechanism of Treg/Th17 imbalance in the pathogenesis of cardiovascular complications for uremic patients undergoing hemodialysis should also be investigated.

\section{References}

1. Carrero JJ, de Jager DJ, Verduijn M, et al: Cardiovascular and noncardiovascular mortality among men and women starting dialysis. Clin J Am Soc Nephrol 6: 1722-1730, 2011.

2. House AA and Ronco C: The burden of cardiovascular risk in chronic kidney disease and dialysis patients (cardiorenal syndrome type 4). Contrib Nephrol 171: 50-56, 2011.

3. Liebman SE, Lamontagne SP, Huang LS, et al: Smoking in dialysis patients: a systematic review and meta-analysis of mortality and cardiovascular morbidity. Am J Kidney Dis 58: 257-265, 2011.

4. Dheir H, Ozkahya M, Kircelli F, et al: Glycosylated hemoglobin levels are associated with cardiovascular events in nondiabetic peritoneal dialysis patients. J Nephrol 25: 107-112, 2012. 
5. Singh AK, Singh V, Pal Singh M, et al: Effect of immunosenescence on the induction of cardiovascular disease pathogenesis: role of peripheral blood mononuclear cells. Immunopharmacol Immunotoxicol 30: 411-423, 2008.

6. Bacharaki D, Thodis E, Passadakis $\mathrm{P}$, et al: Comparative in vitro study of different peritoneal dialysis solutions on cytokine production by peripheral blood mononuclear cells. Nephron Clin Pract 113: c321-c329, 2009.

7. Zhang J, Hua G, Zhang X, et al: Regulatory T cells/T-helper cell 17 functional imbalance in uraemic patients on maintenance haemodialysis: a pivotal link between microinflammation and adverse cardiovascular events. Nephrology 15: 33-41, 2010.

8. Ohkusu-Tsukada K, Toda M, Udono H, et al: Targeted inhibition of IL-10-secreting CD25- Treg via p38 MAPK suppression in cancer immunotherapy. Eur J Immunol 40: 1011-1021, 2010.

9. Korn T, Mitsdoerffer M, Croxford AL, et al: IL-6 controls Th17 immunity in vivo by inhibiting the conversion of conventional $\mathrm{T}$ cells into Foxp3 ${ }^{+}$regulatory T cells. Proc Natl Acad Sci USA 105: 18460-18465, 2008.

10. Xu L, Kitani A, Fuss I and Strober W: Cutting edge: regulatory $\mathrm{T}$ cells induce $\mathrm{CD}^{+}{ }^{+} \mathrm{CD} 25^{-}{ }^{-}$-oxp3 ${ }^{-} \mathrm{T}$ cells or are self-induced to become Th17 cells in the absence of exogenous TGF-beta. J Immunol 178: 6725-6729, 2007.
11. Walker LS: CD4 ${ }^{+}$CD $25^{+}$Treg: divide and rule? Immunology 111: 129-137, 2004.

12. Weaver CT and Hatton RD: Interplay between the TH17 and TReg cell lineages: a (co-)evolutionary perspective. Nat Rev Immunol 9: 883-889, 2009.

13. Chen D, Huang X, Yang M, et al: Treg/Th17 functional disequilibrium in Chinese uremia on hemodialysis: a link between calcification and cardiovascular disease. Ren Fail 34: 697-702, 2012.

14. Haas J, Fritzsching B, Trübswetter P, et al: Prevalence of newly generated naive regulatory $\mathrm{T}$ cells (Treg) is critical for Treg suppressive function and determines Treg dysfunction in multiple sclerosis. J Immunol 79: 1322-1330, 2007.

15. Yamagiwa T, Fukunishi S, Tachibana T, et al: Abrogation of Treg function deteriorates rheumatoid arthritis. Mod Rheumatol 22: 80-88, 2012.

16. Kimura A and Kishimoto T: IL-6: regulator of Treg/Th17 balance. Eur J Immunol 40: 1830-1835, 2010. 